\title{
Identification of a Novel Nucleobase-Ascorbate Transporter Family Member in Fish and Amphibians
}

\author{
Diogo Oliveira ${ }^{1,+}\left(\mathbb{C}\right.$, André M. Machado ${ }^{1,+}+\mathbb{C}$, Tiago Cardoso ${ }^{1}\left(\mathbb{C}\right.$, Mónica Lopes-Marques ${ }^{1}$, \\ L. Filipe C. Castro ${ }^{1,2, *}$ and Raquel Ruivo ${ }^{1, * \mathbb{D}}$ \\ 1 CIIMAR - Interdisciplinary Centre of Marine and Environmental Research, U. Porto-University of Porto, \\ Terminal de Cruzeiros do Porto de Leixões, Av. General Norton de Matos s/n, 4450-208 Matosinhos, \\ Portugal; up201207827@fc.up.pt (D.O.); andre.machado@ciimar.up.pt (A.M.M.); \\ tiago12525@gmail.com (T.C.); monicaslm@hotmail.com (M.L.-M.) \\ 2 Department of Biology, Faculty of Sciences, U. Porto-University of Porto, Rua do Campo Alegre 1021/1055, \\ 4169-007 Porto, Portugal \\ * Correspondence: filipe.castro@ciimar.up.pt (L.F.C.C.); rruivo@ciimar.up.pt (R.R.); \\ Tel.: +351-223-401-800 (R.R.) \\ + These authors contributed equally to this work.
}

Received: 24 October 2018; Accepted: 18 December 2018; Published: 1 January 2019

\begin{abstract}
Nucleobase-Ascorbate Transporter (NAT) family includes ascorbic acid, nucleobases, and uric acid transporters: With broad evolutionary distribution. In vertebrates, four members have been previously recognized, the ascorbate transporters Slc23a1 and Slc3a2, the nucleobase transporter Slc23a4 and an orphan transporter Slc23a3. Using phylogenetic and synteny analysis, we identify a fifth member of the vertebrate slc23 complement (slc23a5), present in neopterygians (gars and teleosts) and amphibians, and clarify the evolutionary relationships between the novel gene and known slc23 genes. Further comparative analysis puts forward uric acid as the preferred substrate for Slc23a5. Gene expression quantification, using available transcriptomic data, suggests kidney and testis as major expression sites in Xenopus tropicalis (western clawed frog) and Danio rerio (zebrafish). Additional expression in brain was detected in D. rerio, while in the Neoteleostei Oryzias latipes (medaka) slc23a5 expression is restricted to the brain. The biological relevance of the retention of an extra transporter in fish and amphibians is discussed.
\end{abstract}

Keywords: Nucleobase-Ascorbate Transporters (NAT); Slc23; Slc23a5; uric acid; antioxidant

\section{Introduction}

Ascorbic acid (or its salt form ascorbate) is an essential enzyme cofactor, participating in collagen and norepinephrine synthesis, as well as a potent antioxidant and free radical scavenger [1,2]. Despite its importance, anthropoid primates, teleost fish, Guinea pigs, some bats, and Passeriformes bird species, lack a functional L-gulono-Y-lactone oxidase (GLO) gene and, thus, the ability to catalyze the last step of ascorbic acid synthesis [3,4]. The scattered loss of the GLO gene was suggested to evolve neutrally, with ascorbic acid requirements being met by dietary intake [3]. Unlike ascorbate, uric acid is largely regarded as end-product of purine metabolism and metabolic waste [5]. Yet, human-biased research has suggested alternative physiological functions for uric acid or urate (uric acid salts), such as antioxidant and free radical scavenger, similarly to ascorbic acid, counterbalancing disease-related oxidative stress [6-8].

Ascorbic and uric acid serve as substrates to active transporters that belong to the Nucleobase-Ascorbate Transporter (NAT) family; NAT family members, which include solute carrier family 23 (Slc23) transporters, display a very broad evolutionary distribution, from bacteria to 
metazoans [1]. Sodium-dependent Slc23 transporters were suggested to maintain vertebrate ascorbic acid levels: With Slc23a1 responsible for renal and intestine ascorbic acid absorption across brush border epithelia and Slc23a2, which exhibits a widespread expression, responsible for ascorbic acid supply to target tissues and cells, including neurons $[1,9,10]$. In vertebrates, two additional transporters were identified, the orphan transporter Slc23a3 and the nucleobase transporter Slc23a4, described in rats but found pseudogenized in humans [1,11]. Rat Slc23a4 is restricted to the small intestine and was shown to predominantly mediate sodium-dependent uracil transport [11]. Slc23a3, on the other hand, is expressed in proximal renal tubules but is unresponsive to both ascorbic acid and nucleobases [1].

NAT family members display a highly conserved signature motif: [Q/E/P]-N-x-G-x-x-x-x-T$[R / K / G][1,11-13]$. Functional characterization of a distinct member of the NAT family highlighted the importance of the first amino acid position of the motif in the definition of substrate specificity [1,11-13]. Thus, these transporters can be divided into three functional groups. Slc23a1, Slc23a2, and Slc23a3 belong to the ascorbate group and harbor a conserved proline $(\mathrm{P})$ in the first amino acid position of the signature motif [1]. Glutamate (E) residue defines the uracil group, including bacterial anion symporters and Slc23a4, while glutamine (Q) is present in the bacterial uric acid and/or xanthine anion symporters [1,11-13]. Topology predictions, using proteins from the distinct groups, suggest a cytosolic location of the motif $[1,11,12]$. Here we revise the portfolio of NAT family members in vertebrates.

\section{Materials and Methods}

\subsection{Sequence Mining}

Slc23 protein sequences were obtained from the GenBank database by BLASTp using human Slc23a1 (NP_005838), Slc23a2 (NP_976072), and Slc23a3 (NP_001138362) sequences as query. The sequences from Lethenteron japonica (Japanese lamprey) were obtained from the Japanese Lamprey Genome Project (http:/ /jlampreygenome.imcb.a-star.edu.sg/). The sequences from Leucoraja erinacea (little skate) and Scyliorhinus canicula (small-spotted catshark) were obtained as nucleotide sequences from SkateBase (http://skatebase.org) and translated. Accession numbers for all the retrieved sequences are listed in Supplementary Table S1.

\subsection{Synteny}

Slc23 genes were mapped onto the respective species genomes, using the latest genome assemblies available in the GenBank database (https: / /www.ncbi.nlm.nih.gov/). Genes flanking Slc23a1, Slc23a2, Slc23a3, Slc23a4 and the novel Slc23a-like gene were identified and mapped using the human loci as a reference. If the target gene was not found, the syntenic genomic region was retrieved using neighboring genes as a reference.

\subsection{Phylogenetic Analysis}

The retrieved amino acid sequences were aligned using the MAFFT software web service (http: / / mafft.cbrc.jp/alignment/software/) in default automatic settings, with the L-INS-I refinement method [14]. The alignment was stripped from columns with at least $20 \%$ of gaps resulting in alignment with 75 sequences and 526 positions. The output alignment was used to construct a phylogenetic tree using PhyML 3.0 with Smart Model Selection web service (http: / / www.atgc-montpellier.fr/phymlsms /) in default settings, which selected the LG $+\mathrm{G}+\mathrm{I}+\mathrm{F}$ model [15]. Bayesian-like transformation of aLRT was selected to assess branch support. An additional phylogenetic tree, with automatic bootstrapping (552 bootstraps) and using the default hill-climbing algorithm, was constructed with RAxML, available in CIPRES Science Gateway.

\subsection{Membrane Topology Prediction}

Slc23a5 membrane topology predictions were performed for selected species, using distinct prediction web servers: TMHMM Server v. 2.0 (http:/ / www.cbs.dtu.dk/services/TMHMM/) and 
TMPred (https://embnet.vital-it.ch/software/TMPRED_form.html). Human Slc23a1 was used as a control.

\subsection{Filtering and Quality Control of Sequence Reading Archive (SRA) Datasets}

For RNA-seq analysis, we included 39 SRA datasets from brain, intestine, kidney, liver, and testis tissues of Homo sapiens (human), Mus musculus (mouse), Gallus gallus (chicken), Anolis carolinensis (American green anole), Xenopus tropicalis (western clawed frog), Danio rerio (zebrafish), Lepisosteus oculatus (spotted gar), and Oryzias latipes (Japanese medaka), which were obtained from the National Center for Biotechnology (NCBI) Sequence Read Archive (SRA) (https:/ /www.ncbi.nlm.nih.gov/sra/) (Table S2). The read quality assessment of each SRA dataset was performed using FastQC software (https://www.bioinformatics.Babraham.ac.uk/projects/fastqc/). Trimmomatic 0.36 [16] was used to trim and drop reads with quality scores below 5 , at leading and trailing ends, with an average quality score below 20 in a 4 bp sliding window and with less than 36 bases length.

\subsection{Relative Gene Expression Levels}

To assess the relative gene expression of slc23 genes from the selected species, reference sequences and the corresponding annotations were collected from NCBI and Ensembl [17] databases. For human, mouse, lizard, zebrafish and spotted gar, the GTF and DNA files (genome) were retrieved from Ensembl (Release 89) [17]. For chicken and western clawed frog, transcript sequences (coding and non-coding) and mapping (gene/transcript) from NCBI Refseq database were used after filtering [18] (for each species the rna.fasta file from ftp:/ / ftp.ncbi.nih.gov/genomes/refseq/ was used-See Tables S3 and S4 for detail). The SRA reads of each specimen were aligned to the reference using Bowtie2 (default parameters) [19], and gene expression was quantified as transcript per million (TPM) using RSEM v.1.2.31 (RNA-seq by Expectation Maximization) software package [20]. Isoform expression levels for each gene were summed to derive the TPM values. For genes with low relative expression values, TPM values were log2-transformed after adding a value of one; TPM values $<0.5$ were considered unreliable and substituted with zero.

\subsection{PCR-Based Validation of Slc23a5 Expression}

Total RNA extraction from $X$. tropicalis and D. rerio tissues was performed using the Illustra RNAspin Mini RNA Isolation Kit animal tissues protocol with on-column DNAseI digestion (GE Healthcare, Chicago, IL, USA). RNA quality was visually assessed by electrophoresis and sample concentration was determined using the Take3 micro-volume plate system (BioTek, Winooski, VT, USA). First-strand cDNA was synthesized from $250 \mathrm{ng}$ of total RNA using the iScriptTM cDNA Synthesis Kit (Bio-Rad, Hercules, CA, USA), according to the manufacturer's instructions. Gene expression of $S l c 23 a 5$ was analyzed by PCR using the intro flanking expression primer pairs $5^{\prime}$-AGACGTTGAAGCCGTTACTGA- $3^{\prime}$ and $5^{\prime}$-TGCTATTATGCCTCCAAAGGCT- $3^{\prime}$ for X. tropicalis and $5^{\prime}$-GGTGGAATGTTCCTCGTCAT- $3^{\prime}$ and $5^{\prime}$-GATGAACATGTGGGTGGTGA-3' for $D$. rerio. PCR reactions were carried out on a tissue panel containing heart, skin, stomach, intestine, liver, kidney, ovary, and testis tissues for X. tropicalis and brain, heart, skin, gut, liver, kidney, ovary, and testis tissues for D. rerio. The PCR was performed with Phusion ${ }^{\circledR}$ Flash high fidelity Master Mix (Thermofisher, Waltham, MA, USA), $1 \mu \mathrm{M}$ of each primer and $25 \mathrm{ng} / \mu \mathrm{L}$ of template cDNA, with the following parameters: Initial denaturation at $98{ }^{\circ} \mathrm{C}$ for $1 \mathrm{~s}$, annealing at $58{ }^{\circ} \mathrm{C}$ for $D$. rerio or $66^{\circ} \mathrm{C}$ for $X$. tropicalis primers and elongation at $72{ }^{\circ} \mathrm{C}$ for $4 \mathrm{~s}$, with 30 cycles.

\section{Results}

\subsection{Identification of a Novel Member of the NAT Family in Neopterygians and Amphibians}

Through database mining and phylogenetic analysis, we detected the presence of a novel gene, slc23a5, found in neopterygians (Holostei and Teleostei) and amphibians (Figures 1 and S1). No slc23a5 
sequence was retrieved from chondrichthyans, chondrosteans (basal actinopterygians), nor from the sarcopterygian Latimeria chalumnae (coelacanth). Subsequent loss of slc23a5 in chondrichthyans was further supported by the conservation of the genomic locus in Callorhynchus milii (Australian ghostshark) (Figure S2). Regarding coelacanth, two scaffolds, denoting conservation of the flanking genes, were found; while, no information was retrieved regarding the chondrostean loci (Figure S2). The remaining genes of the slc23 family were retrieved and genomic loci were found generally conserved across vertebrate species, with exception of the slc23a4 loci in fish (Figures S3-S6). The branching pattern of our phylogenetic analysis suggests that after the expansion of the slc23a gene family slc23a5 was subsequently lost in sharks, basal actinopterygians, coelacanth, and amniotes (Figures 1 and S2). In agreement, we obtained three phylogenetic clusters possibly corresponding to three ancestral lineages (Figures 1 and S1): One composed of typical slc23a1 and slc23a2 genes and a second group including the previously reported slc23a4 and the novel slc23a5 genes, both out-grouped by invertebrate sequences. A third cluster, which outgroups the remaining slc23 genes, included the highly divergent slc23a3 genes, mirrored by the long branch lengths (Figure 1).

\subsection{Slc23a5 Signature Motifs}

Further comparative protein sequence analysis highlighted the presence of a NAT family signature motif with a conserved $\mathrm{Q}$ in the substrate determining position. According to previous functional characterization of NAT family members, this residue is found in uric acid and/or xanthine transporters, thus placing Slc23a5 in the uric acid and/or xanthine group (Figure 2) [1,11-13]. Besides the typical signature motif, a carboxyl terminus tripeptide motif was identified in some SLC23 family members: An S/T-X- $\varnothing$ tripeptide. S/T-X- $\varnothing$ motifs were shown to interact with PDZ domains of scaffolding proteins belonging to the sodium/hydrogen exchanger regulatory factor, NHERF, family [21]. S/T-X-Ø are found highly conserved across species in Slc23a1 and Slc23a4, in agreement with their renal and intestinal expression and role in ascorbic acid and nucleobase absorption (Figure S7). Slc23a5, on the other hand, exhibits a highly conserved tripeptide motif in Otocephala (Cypriniformes, Characiformes, and Siluriformes) but not Osteoglossomorpha (Figure 3A). The Euteleostei Esociformes and Salmoniformes exhibit an inverted motif while in the Neoteleostei no conservation was found. Finally, topology predictions yielded similar transmembrane profiles for Slc23a5 sequences from D. rerio, O. latipes, Salmo salar (Atlantic salmon) and X. tropicalis, when compared to human Slc23a1 (Figure S8). The NAT motif was predicted cytosolic in all analyzed sequences. This suggests a conserved cellular import topology, as observed in other family members. 


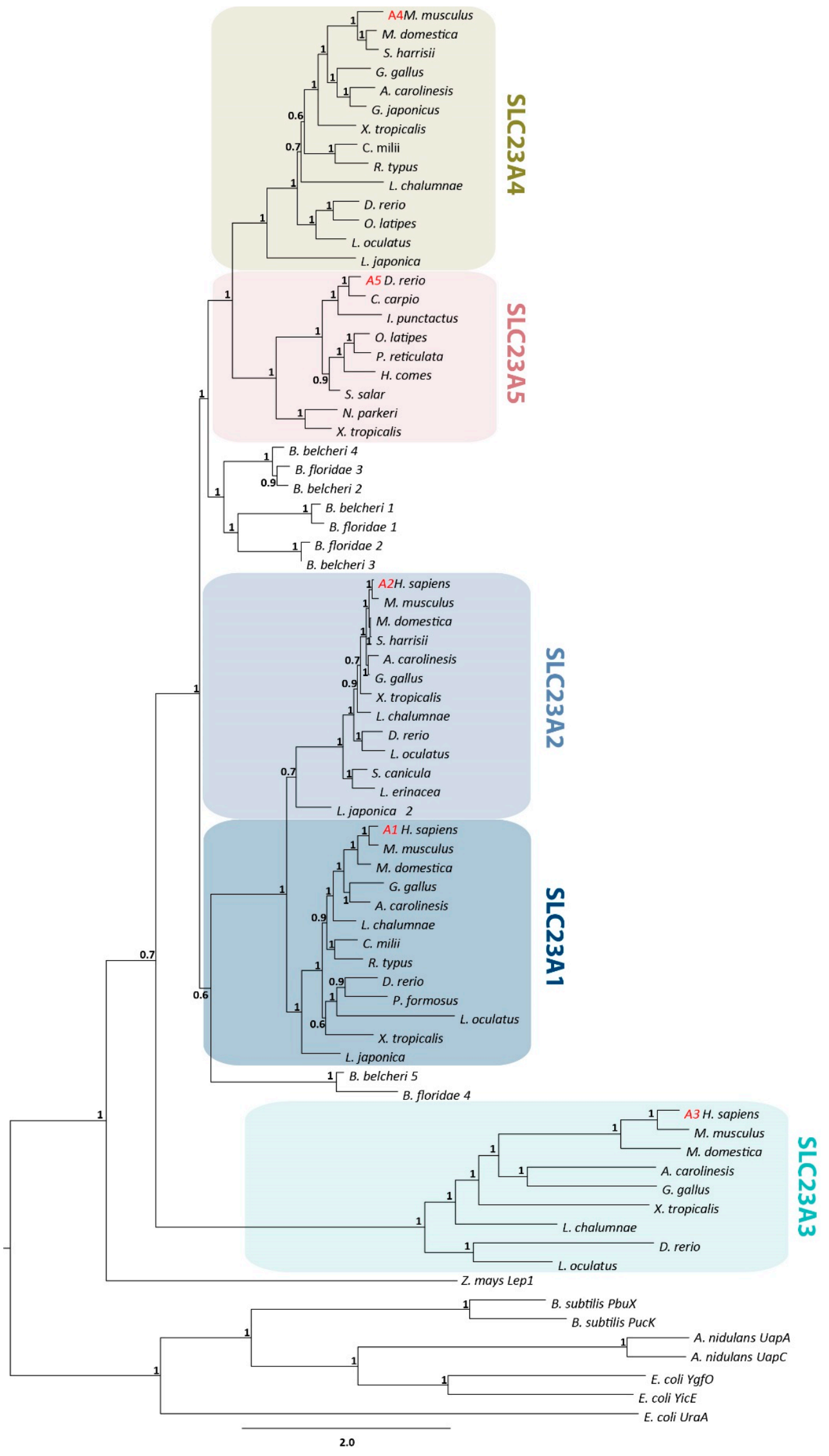

Figure 1. Maximum likelihood phylogenetic tree describing relationships among Nucleobase-Ascorbate Transporter (NAT) family members. Node values represent branch support using the aBayes algorithm. Accession numbers for all sequences are provided in Supplementary Materials. 


\section{Sequence Logo}

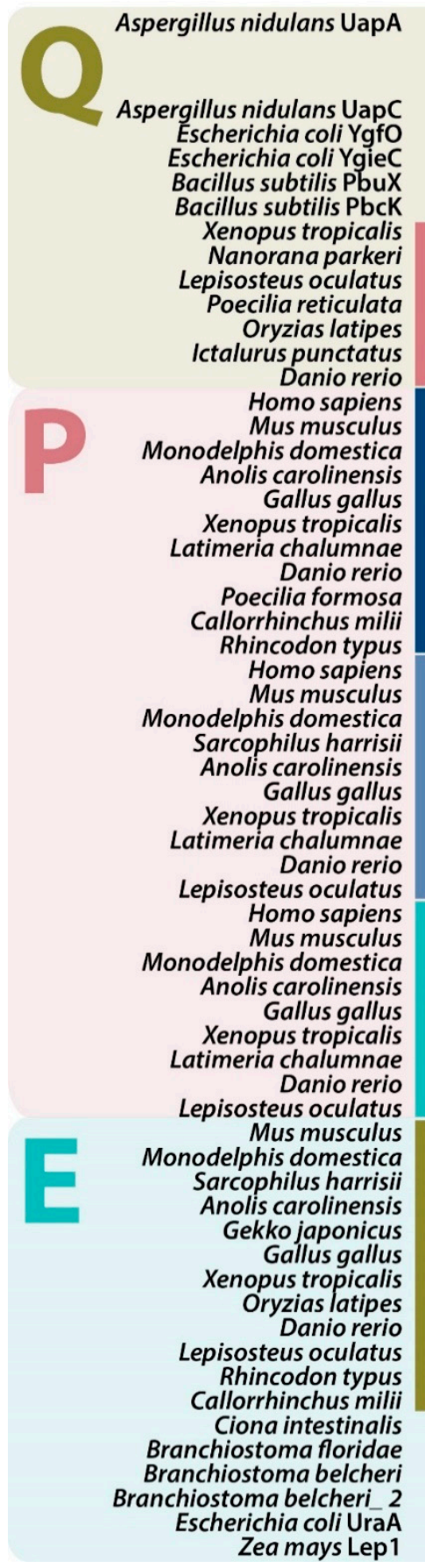

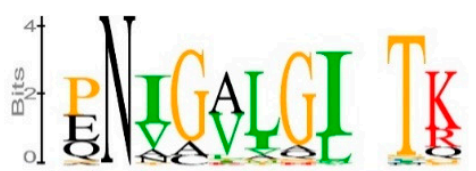

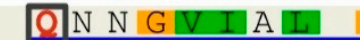

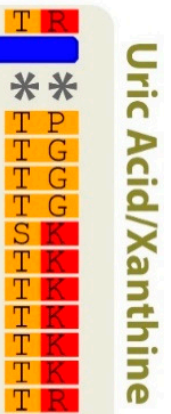

$\frac{7}{\frac{0}{0}} \frac{0}{n}$
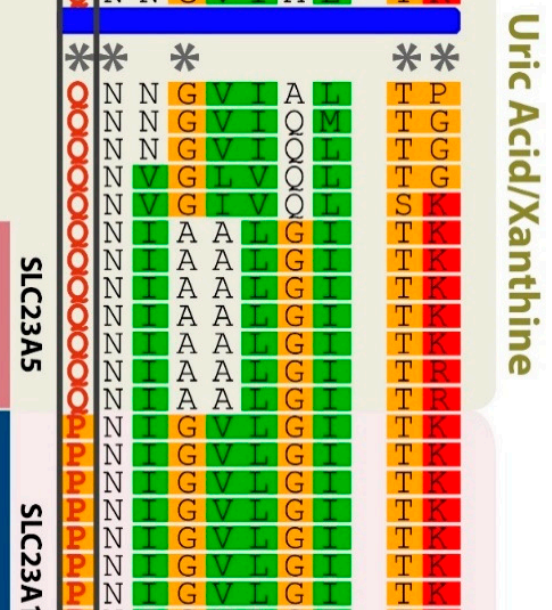

N T G L I I

N I G V L G

N I G G V L G

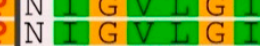

N I G G L G I
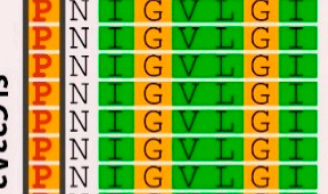

T

T

T

$\mathrm{T}$

五

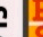

$\checkmark A$ T T S

$\mathrm{T}$

T

w A N A C C A G G L

$\gg$ P N A G L A G I

A C A H G

$\begin{array}{llllll}A & C & V & I & G & I \\ A & C & A & L & G & I\end{array}$

\begin{tabular}{l|l|llllll}
$P$ & $N$ & $A$ & $C$ & $A$ & $L$ & $G$ & $L$ \\
\hline & $N$ & $V$ & $G$ & $A$ & $L$ & $G$ & $I$ \\
\hline & $N$ & $V$ & $G$ & $A$ & $L$ & $G$ & $I$ \\
\hline & $N$ & $V$ & $G$ & $A$ & $L$ & $G$ & $I$ \\
\hline
\end{tabular}

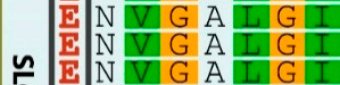

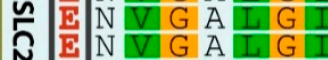

\begin{tabular}{llllllll}
\hline & & $V$ & $G$ & $A$ & $L$ & $G$ & $I$ \\
\hline$N$ & $V$ & $G$ & $A$ & $L$ & $G$ & $I$ \\
\hline
\end{tabular}

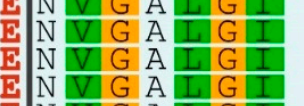

Figure 2. Alignment of the signature motif of the Nucleobase-Ascorbate Transporter (NAT) family. NAT family members display a highly conserved signature motif defining substrate specificity: [Q/E/P]-N-x-G-x-x-x-x-T-[R/K/G]. Asterisks indicate conserved positions. The first amino acid position, with residues highlighted in red within a black box, defines substrate preference: Q-Uric acid/xanthine group; $\mathrm{P}$-Ascorbate group and E-Uracil group. The mirror $\mathrm{Q}$ in the Slc23a3 group is also highlighted by a black box. 
A)

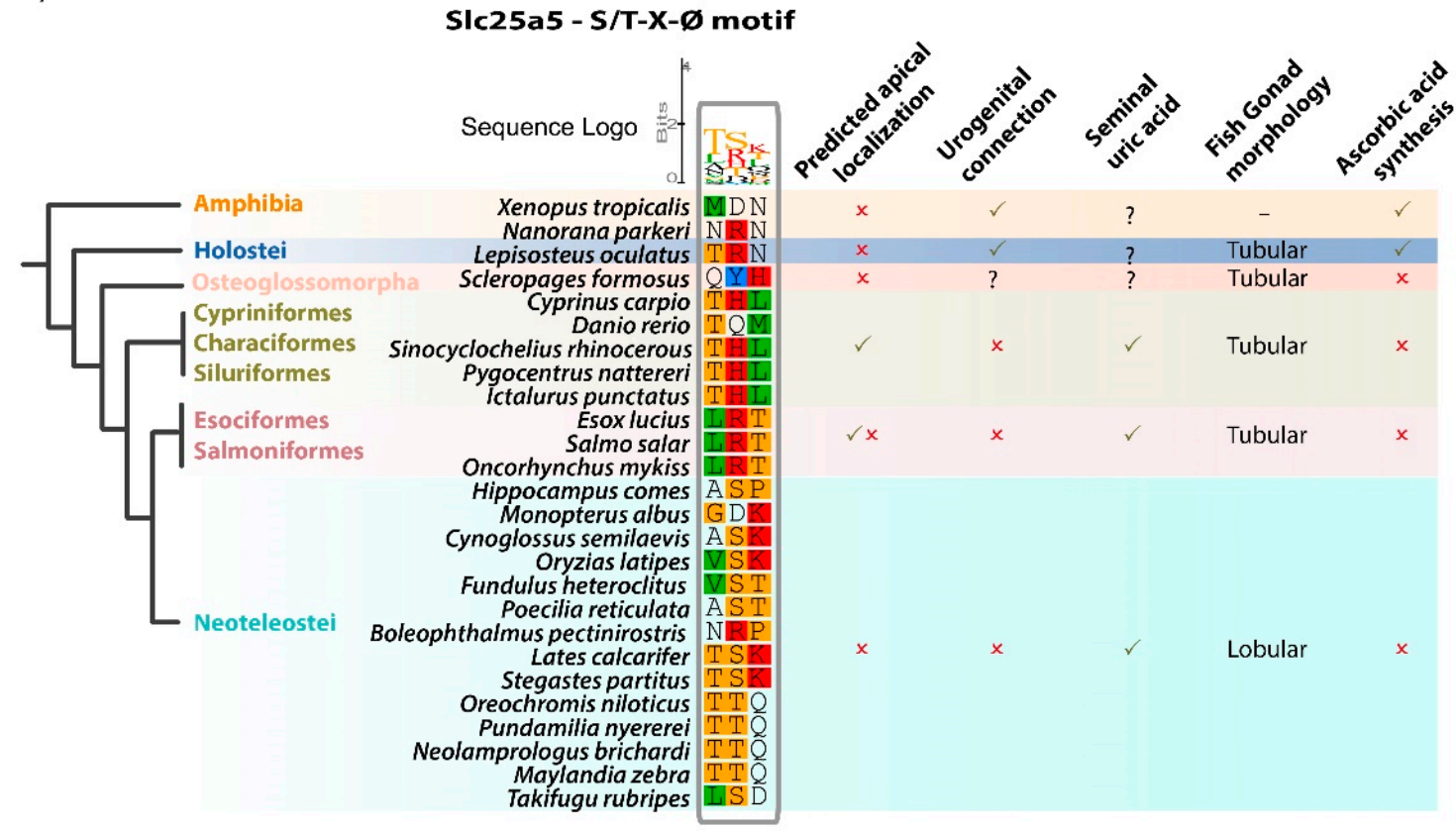

B)

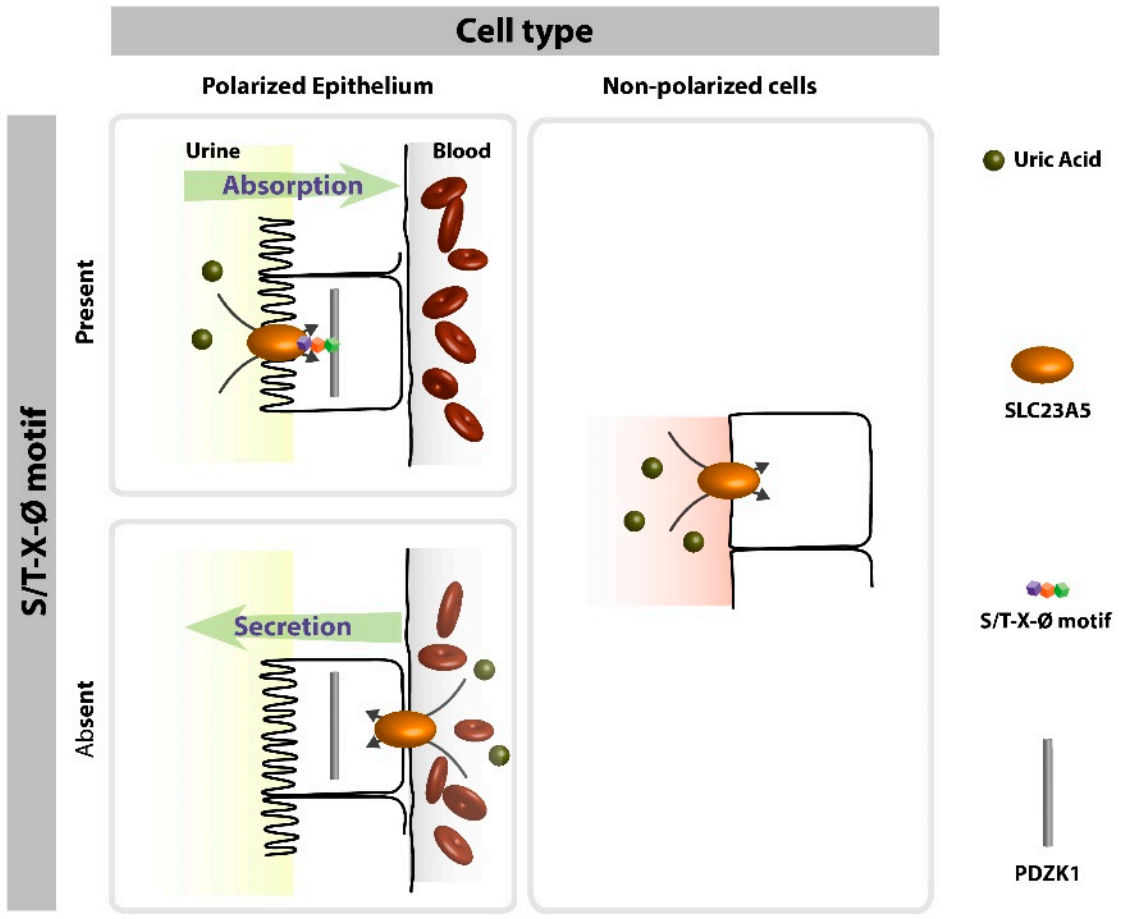

Figure 3. The S/T-X- $\varnothing$ tripeptide provides insight into the cellular function of Slc23a5 homologues from fish and amphibians. (A) Alignment of the PDZ-binding tripeptide in Slc23a5 homologues from fish and amphibians. The S/T-X- $\varnothing$ tripeptide is found in Cypriniformes, Characiformes, and Siluriformes while the Euteleostei Esociformes and Salmoniformes exhibit an inverted motif. Predicted cellular localization is indicated as well as morphological and physiological adaptations among the different fish groups. (B) Schematic representation of tripeptide-dependent uric acid flows in polarized epithelia and non-polarized cells. In polarized epithelia (i.e., kidney) the presence of the tripeptide motif suggests interaction with the scaffolding protein PDZK1 which localizes apically, in the absorptive side. 


\subsection{Slc23a5 Expression Patterns}

To gain further insight into the functional relevance of this additional gene, we collected RNA-seq data and determined relative expression levels of Slc23 genes. Gene expression analysis suggested that in D. rerio and X. tropicalis, slc23a5 is mostly expressed in kidney and testis, further confirmed by PCR (Figure 4 and Table S5). Expression in the brain was also detected in D. rerio and was found to be the exclusive expression site in the Neoteleostei O. latipes. The holostean L. oculatus, on the other hand, yielded no significant expression in the tested tissues.

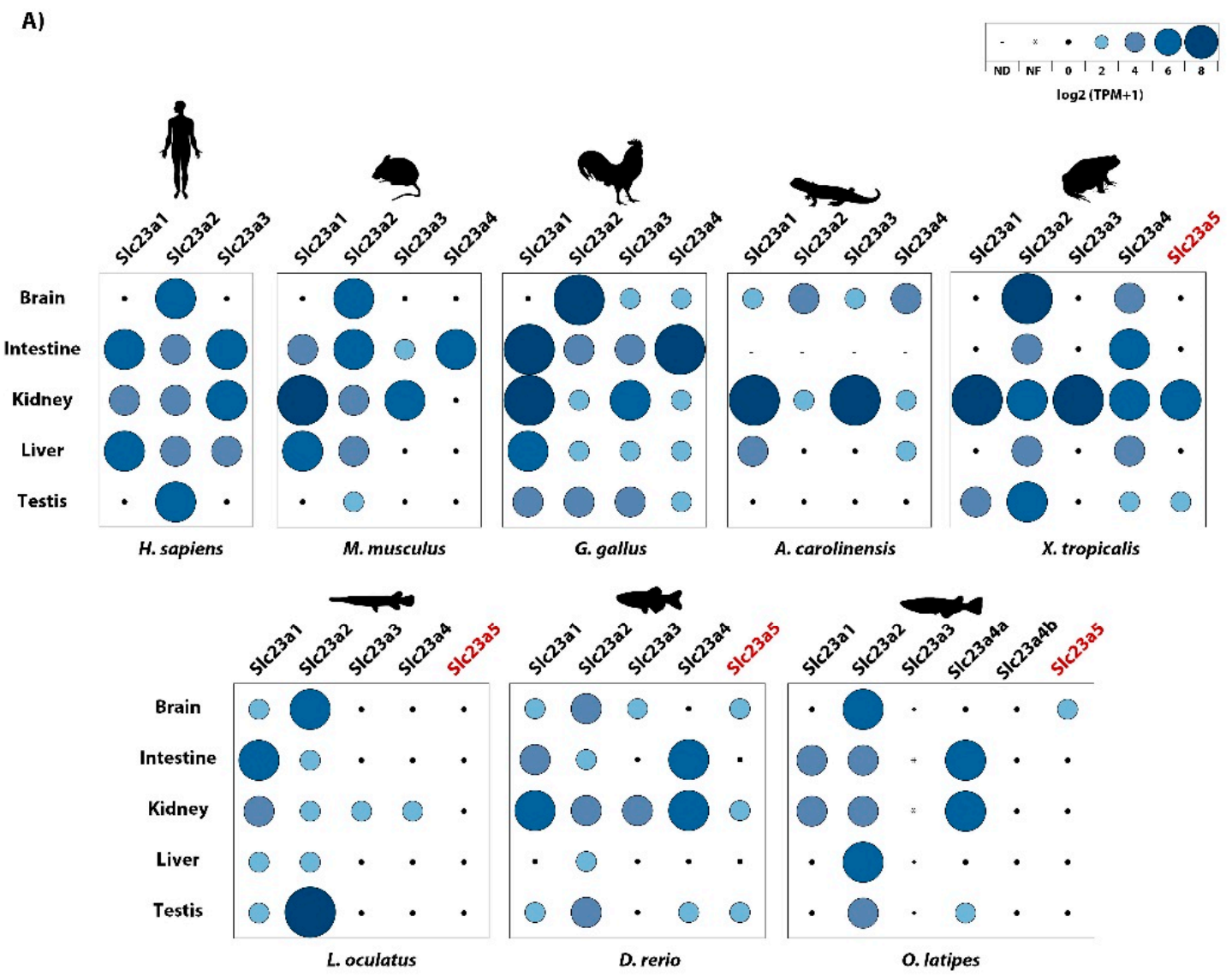

B)

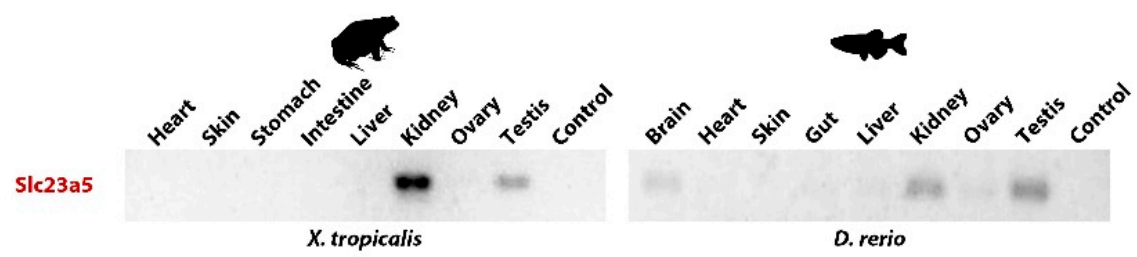

Figure 4. Expression patterns of Slc23 isoforms across vertebrates. (A) RNAseq-based quantification. Relative gene expression levels are provided as $\log _{2}$-transformed transcript per million (TPM) values, from 0 to 8 TPM's, NF - not found, ND - no sequence reading archive (SRA) data available for the specific tissue. (B) PCR-based amplification of Slc23a5 transcripts in D. rerio and X. tropicalis tissues.

\section{Discussion}

Our findings indicate a restricted distribution of slc23a5 to taxa that share an aquatic nature of their habitat. In fact, slc23a5 was found to be expressed in the kidney and testis of both X. tropicalis and D. rerio. In the latter, as well as in the Neoteleostei O. latipes, brain expression was also detected. Comparative analysis using previously characterized NAT family transporters placed this novel transporter in the uric acid and/or xanthine transporter group. In fact, similarly to uric acid and/or 
xanthine transporters, Slc23a5 proteins harbor a $Q$ in the first position of the NAT family signature motif ([Q/E/P]-N-x-G-x-x-x-x-T-[R/K/G]). Yet, further functional studies are required to fully clarify the molecular function of Slc23a5 proteins, notably its uric acid and/or xanthine binding abilities.

Given the nature of NAT family transporter ligands, including ascorbic and uric acids, xanthine or nucleobases, renal Slc23a5 could participate in the homeostatic maintenance of excretion products, in osmotic regulation, and/or in antioxidant processes. In fact, kidneys play a crucial role in the maintenance of both ascorbic and uric acid levels through the excretion and reabsorptions processes [6,21]. In kidney absorptive epithelium, transporters located on the filtrate-epithelial interface (apical side) interact with the scaffolding protein PDZ-containing kidney protein 1 (PDZK1) [21]. PDZK1 belongs to the NHERF family of proteins which are mostly expressed in the apical side of absorptive epithelia of the kidney, intestine, and liver, and establish protein-protein interaction networks with receptors, transporters, and channels [22,23]. Besides its scaffolding role, NHERF family members were also shown to modulate the activity of protein assemblages [23-25]. Within Slc23 family members, S/T-X- $\varnothing$ motifs are found in Slc23a1 and Slc23a4 proteins, which are present in renal and intestinal polarized epithelia and participate in ascorbic acid and nucleobase absorption, and absent in Slc23a2. Curiously, a highly conserved PDZK1 interacting motif (S/T-X-Ø) was also detected in Slc23a5 from Cypriniformes, Characiformes, and Siluriformes, but not in other fish groups or amphibians. No relationship between salt and freshwater environments was found. Thus, the presence/absence of this peptide signal might reflect distinct flows in the kidney: An apically expressed Slc23a5 likely participates in absorptive flows, while a basolateral expression would be related to secretion (Figure 3B).

In the human proximal renal tubules, several transporters, localized to specific cellular regions (apical or basolateral), seem to cooperate for efficient urate handling: Named the urate transportome [6,21]. Thus, if the predicted uric acid transport activity is confirmed, X. tropicalis and D. rerio Slc23a5 could illustrate the two opposing mechanisms: While D. rerio captures, $X$. tropicalis secretes uric acid into the renal filtrate. In agreement, X. tropicalis, D. rerio, as well as L. oculatus, appear to lack the full complement of renal urate transporters (Table S6). A similar scenario is observed for birds and reptiles; however, their excretory system represents a particular adaptation for uric acid excretion $[26,27]$. Besides simple excretion, and given that high concentrations of uric acid were detected, and suggested to act as the main non-enzymatic antioxidant mechanism, in seminal fluid of several fish species (including Cypriniformes, Esociformes, Salmoniformes, and the Neoteleostei Gadiformes and Perciformes [28-31]), this putative uric acid transport activity could be maintained for reproductive purposes, in agreement with the observed testicular expression. In fact, in external fertilizers, such as most fish and amphibians, reactive oxygen species were suggested to decrease motility and thus fertilization success [30]. Yet, regarding amphibians, no information on seminal fluid composition was retrieved [32]. Nonetheless, in vitro assays suggested that other antioxidant species, such as ascorbic acid or vitamin E, were unsuitable at offsetting oxidative stress and improving sperm motility in Litoria booroolongensis (Booroolong frog) and suggested uric acid as a pertinent candidate [32]. In this context, the absence of slc23a5 sequences in Chondrichthyes could be due to their internal fertilization strategies derived from ovoviviparous or viviparous reproductive modes [33]. Accordingly, viviparity is also observed in the sarcopterygian L. chalumnae [33].

Besides reproductive categories, other morphological and metabolic traits could justify the seemingly scattered retention of slc23a5 among fish in the context of the proposed uric acid transport activity; for instance, specific anatomical features such as the presence of a urogenital connection: Described in Elasmobranchii and in most non-teleost Osteichthyes, yet absent in Cyclostomata and Teleostei [34]. By connecting testicular and renal ducts, antioxidant requirements could be met by renal secretion into the urine. In agreement, in the Chondrostei Acipenser ruthenus, full sperm maturation and motility were shown to require dilution by urine [35]. A urogenital connection is also present in amphibians [34]. Additionally, other antioxidant mechanisms could exist. Besides uric acid, ascorbic acid is also present in the seminal fluid of Oncorhynchus mykiss (rainbow trout) [36]. Seminal ascorbate was reported to be affected by seasonal variations and semen quality was shown to improve upon 
dietary ascorbic acid supplementation [36,37], contrasting with the amphibian L. booroolongensis [32]. In the case of non-teleost fish, such as Chondrostei (A. ruthenus) and Holostei (L. oculatus), a functional GLO gene is retained, providing endogenously synthesized ascorbate [38]. Curiously, no gonadal expression was detected in the Neoteleostei O. latipes. Neoteleosts exhibit varied reproduction modes ranging from external fertilizers to internal fertilizers with viviparity [33,39-41]. In addition, neoteleosts were shown to exhibit a distinctive gonadal morphology, the lobular testis, whereas the remaining bony fish exhibit tubular testis [42]. Although still obscure, the absence of testicular slc23a5 expression in the external fertilizer $O$. latipes could be due to specific morphological adaptations of neoteleosts.

A similar role could be postulated for neuronal uric acid [43]. Active swimming behavior requires a high metabolic rate, mirrored by increased oxygen consumption, subsequently leading to reactive oxygen species production [44]. In agreement, fish antioxidant levels were shown to be correlated with their swimming activity [44]. Neuronal cell populations are particularly sensitive to oxidative stress, thus, uric acid could participate in the neutralization of oxidative species to by-pass oxidative stress, notably in GLO-deficient fish. Yet, information on neuronal uric acid levels in fish is currently absent.

\section{Conclusions}

Taken together, these observations put forward the retention of an additional Slc23 gene, in neopterygians and amphibians, with restricted expression patterns and presumed uric acid transport function. Yet, the extensive comparative functional analysis should be conducted to clarify the physiological relevance of this novel receptor in aquatic organisms.

Supplementary Materials: The following are available online at http:/ /www.mdpi.com/2410-3888/4/1/1/s1, Supplementary tables and figures including synteny, phylogeny and topology analysis, as well as reference tables.

Author Contributions: Conceptualization, R.R. and L.F.C.C.; Validation, R.R.; Formal analysis, D.O, A.M.M. and R.R.; Investigation, D.O., A.M.M., M.L.-M. and T.C.; Resources, R.R. and L.F.C.C.; Writing-original draft, R.R., D.O., A.M.M., T.C., M.L.-M. and L.F.C.C.; Writing-review \& editing, R.R. and L.F.C.C.; Visualization, R.R., D.O. and A.M.M.; Supervision, L.F.C.C. and R.R.; Project administration, L.F.C.C.; Funding acquisition, L.F.C.C.

Funding: This research was funded by Coral-Sustainable Ocean Exploitation (Norte-01-0145-FEDER-000036), a project by the North Portugal Regional Operational Program (NORTE 2020), under the PORTUGAL 2020 Partnership Agreement, through the European Regional Development Fund (ERDF) and by the European Regional Development Fund (ERDF) through the COMPETE-Operational Competitiveness Programme and POPH-Operational Human Potential Programme.

Conflicts of Interest: The authors declare no conflicts of interest.

\section{References}

1. Burzle, M.; Suzuki, Y.; Ackermann, D.; Miyazaki, H.; Maeda, N.; Clemencon, B.; Burrier, R.; Hediger, M.A. The sodium-dependent ascorbic acid transporter family SLC23. Mol. Aspects Med. 2013, 34, 436-454. [CrossRef]

2. Linster, C.L.; Van Schaftingen, E. Vitamin C. FEBS J. 2007, 274, 1-22. [CrossRef] [PubMed]

3. Drouin, G.; Godin, J.R.; Page, B. The genetics of vitamin C loss in vertebrates. Curr. Genomics 2011, 12, 371-378. [CrossRef] [PubMed]

4. Dutta Gupta, S.; Choudhury, P.K.; Chatterjee, I.B. Synthesis of 1-ascorbic acid from d-glucurono-1,4-lactone conjugates by different species of animals. Int. J. Biochem. 1973, 4, 309-314. [CrossRef]

5. Wright, P.A. Nitrogen excretion: Three end products, many physiological roles. J. Exp. Biol. 1995, 198, 273-281. [PubMed]

6. Bobulescu, I.A.; Moe, O.W. Renal transport of uric acid: Evolving concepts and uncertainties. Adv. Chronic Kidney Dis. 2012, 19, 358-371. [CrossRef] [PubMed]

7. Alvarez-Lario, B.; Macarrón-Vicente, J. Evolution of Uric Acid Metabolism in Humans. In eLS; John Wiley \& Sons, Ltd.: Hoboken, NJ, USA, 2001. [CrossRef]

8. Oda, M.; Satta, Y.; Takenaka, O.; Takahata, N. Loss of urate oxidase activity in hominoids and its evolutionary implications. Mol. Biol. Evol. 2002, 19, 640-653. [CrossRef] [PubMed] 
9. Corti, A.; Casini, A.F.; Pompella, A. Cellular pathways for transport and efflux of ascorbate and dehydroascorbate. Arch. Biochem. Biophys. 2010, 500, 107-115. [CrossRef]

10. Savini, I.; Rossi, A.; Pierro, C.; Avigliano, L.; Catani, M.V. SVCT1 and SVCT2: Key proteins for vitamin C uptake. Amino Acids 2008, 34, 347-355. [CrossRef]

11. Yamamoto, S.; Inoue, K.; Murata, T.; Kamigaso, S.; Yasujima, T.; Maeda, J.Y.; Yoshida, Y.; Ohta, K.Y.; Yuasa, H. Identification and functional characterization of the first nucleobase transporter in mammals: Implication in the species difference in the intestinal absorption mechanism of nucleobases and their analogs between higher primates and other mammals. J. Biol. Chem. 2010, 285, 6522-6531. [CrossRef]

12. Koukaki, M.; Vlanti, A.; Goudela, S.; Pantazopoulou, A.; Gioule, H.; Tournaviti, S.; Diallinas, G. The nucleobase-ascorbate transporter (NAT) signature motif in UapA defines the function of the purine translocation pathway. J. Mol. Biol. 2005, 350, 499-513. [CrossRef] [PubMed]

13. Papakostas, K.; Frillingos, S. Substrate selectivity of YgfU, a uric acid transporter from Escherichia coli. J. Biol. Chem. 2012, 287, 15684-15695. [CrossRef] [PubMed]

14. Katoh, K.; Standley, D.M. MAFFT multiple sequence alignment software version 7: Improvements in performance and usability. Mol. Biol. Evol. 2013, 30, 772-780. [CrossRef] [PubMed]

15. Guindon, S.; Dufayard, J.F.; Lefort, V.; Anisimova, M.; Hordijk, W.; Gascuel, O. New algorithms and methods to estimate maximum-likelihood phylogenies: Assessing the performance of PhyML 3.0. Syst. Biol. 2010, 59, 307-321. [CrossRef]

16. Bolger, A.M.; Lohse, M.; Usadel, B. Trimmomatic: A flexible trimmer for Illumina sequence data. Bioinformatics 2014, 30, 2114-2120. [CrossRef] [PubMed]

17. Yates, A.; Akanni, W.; Amode, M.R.; Barrell, D.; Billis, K.; Carvalho-Silva, D.; Cummins, C.; Clapham, P.; Fitzgerald, S.; Gil, L.; et al. Ensembl 2016. Nucleic Acids Res. 2016, 44, D710-D716. [CrossRef] [PubMed]

18. O’Leary, N.A.; Wright, M.W.; Brister, J.R.; Ciufo, S.; Haddad, D.; McVeigh, R.; Rajput, B.; Robbertse, B.; Smith-White, B.; Ako-Adjei, D.; et al. Reference sequence (RefSeq) database at NCBI: Current status, taxonomic expansion, and functional annotation. Nucleic Acids Res. 2016, 44, D733-D745. [CrossRef]

19. Langmead, B.; Salzberg, S.L. Fast gapped-read alignment with Bowtie 2. Nat. Methods 2012, 9, 357-359. [CrossRef]

20. Li, B.; Dewey, C.N. RSEM: Accurate transcript quantification from RNA-Seq data with or without a reference genome. BMC Bioinformatics 2011, 12, 323. [CrossRef]

21. Anzai, N.; Jutabha, P.; Amonpatumrat-Takahashi, S.; Sakurai, H. Recent advances in renal urate transport: Characterization of candidate transporters indicated by genome-wide association studies. Clin. Exp. Nephrol. 2012, 16, 89-95. [CrossRef]

22. Dev, K.K. Making protein interactions druggable: Targeting PDZ domains. Nat. Rev. Drug Discov. 2004, 3, 1047-1056. [CrossRef] [PubMed]

23. Clapéron, A.; Mergey, M.; Fouassier, L. Roles of the scaffolding proteins NHERF in liver biology. Clin. Res. Hepatol. Gastroenterol. 2011, 35, 176-181. [CrossRef] [PubMed]

24. Raghuram, V.; Mak, D.-O.D.; Foskett, J.K. Regulation of cystic fibrosis transmembrane conductance regulator single-channel gating by bivalent PDZ-domain-mediated interaction. Proc. Natl. Acad. Sci. USA 2001, 98, 1300-1305. [CrossRef] [PubMed]

25. Wang, S.; Yue, H.; Derin, R.B.; Guggino, W.B.; Li, M. Accessory Protein Facilitated CFTR-CFTR Interaction, a Molecular Mechanism to Potentiate the Chloride Channel Activity. Cell 2000, 103, 169-179. [CrossRef]

26. Braun, E.J. Comparative renal function in reptiles, birds, and mammals. Semin. Avian Exotic. Pet. Med. 1998, 7, 62-71. [CrossRef]

27. Singer, M.A. Do mammals, birds, reptiles and fish have similar nitrogen conserving systems? Comp. Biochem. Physiol. B Biochem. Mol. Biol. 2003, 134, 543-558. [CrossRef]

28. Ciereszko, A.; Dabrowski, K.; Kucharczyk, D.; Dobosz, S.; Goryczko, K.; Glogowski, J. The presence of uric acid, an antioxidantive substance, in fish seminal plasma. Fish Physiol. Biochem. 1999, 21, 313-315. [CrossRef]

29. Dzyuba, V.; Dzyuba, B.; Cosson, J.; Boryshpolets, S.; Yamaner, G.; Kholodniy, V.; Rodina, M. The antioxidant system of sterlet seminal fluid in testes and Wolffian ducts. Fish Physiol. Biochem. 2014, 40, 1731-1739. [CrossRef]

30. Lahnsteiner, F.; Mansour, N. A comparative study on antioxidant systems in semen of species of the Percidae, Salmonidae, Cyprinidae, and Lotidae for improving semen storage techniques. Aquaculture 2010, 307, 130-140. [CrossRef] 
31. Lahnsteiner, F.; Mansour, N.; Plaetzer, K. Antioxidant systems of brown trout (Salmo trutta f. fario) semen. Anim. Reprod. Sci. 2010, 119, 314-321. [CrossRef]

32. Keogh, L.M.; Byrne, P.G.; Silla, A.J. The effect of antioxidants on sperm motility activation in the Booroolong frog. Anim. Reprod. Sci. 2017, 183, 126-131. [CrossRef] [PubMed]

33. Goodwin, N.B.; Dulvy, N.K.; Reynolds, J.D. Life-history correlates of the evolution of live bearing in fishes. Philos. Trans. R. Soc. Lond. B. Biol. Sci 2002, 357, 259-267. [CrossRef]

34. Blün, V. Comparative Anatomy of the Urogenital System. In Vertebrate Reproduction, a Textbook; Springer: Berlin/Heidelberg, Germany; New York, NY, USA; Tokyo, Japan, 1986; pp. 43-74. [CrossRef]

35. Dzyuba, B.; Cosson, J.; Boryshpolets, S.; Bondarenko, O.; Dzyuba, V.; Prokopchuk, G.; Gazo, I.; Rodina, M.; Linhart, O. In vitro sperm maturation in sterlet, Acipenser ruthenus. Reprod. Biol. 2014, 14, 160-163. [CrossRef] [PubMed]

36. Ciereszko, A.; Dabrowski, K. Sperm quality and ascorbic acid concentration in rainbow trout semen are affected by dietary vitamin C: An across-season study. Biol. Reprod. 1995, 52, 982-988. [CrossRef] [PubMed]

37. Ciereszko, A.; Dabrowski, K. Effect of ascorbic acid supplement in vitro onrainbow trout sperm viability. Aquacult. Int. 2000, 8, 1-8. [CrossRef]

38. Moreau, R.; Dabrowski, K. Biosynthesis of ascorbic acid by extant actinopterygians. J. Fish Biol. 2000, 57, 733-745. [CrossRef]

39. Forsman, A.; Tibblin, P.; Berggren, H.; Nordahl, O.; Koch-Schmidt, P.; Larsson, P. Pike Esox lucius as an emerging model organism for studies in ecology and evolutionary biology: A review. J. Fish Biol. 2015, 87, 472-479. [CrossRef]

40. Bone, Q.; Moore, R.H. Biology of Fishes; Taylor \& Francis Group: New York, NY, USA, 2008.

41. Scott, D.B.C.; Fuller, J.D. The reproductive biology of Scleropages formosus (Müller \& Schlegel) (Osteoglossomorpha, Osteoglossidae) in Malaya, and the morphology of its pituitary gland. J. Fish Biol. 1976, 8, 45-53. [CrossRef]

42. Parenti, L.R.; Grier, H.J. Evolution and phylogeny of gonad morphology in bony fishes. Integr. Comp. Biol. 2004, 44, 333-348. [CrossRef]

43. Uttara, B.; Singh, A.V.; Zamboni, P.; Mahajan, R.T. Oxidative Stress and Neurodegenerative Diseases: A Review of Upstream and Downstream Antioxidant Therapeutic Options. Curr. Neuropharmacol. 2009, 7, 65-74. [CrossRef]

44. Lopez-Cruz, R.I.; Dafre, A.L.; Filho, D.W. Oxidative Stress in Sharks and Rays. In Oxidative Stress in Aquatic Ecosystems; Abele, D., Vazquez-Medina, J.P., Zenteno-Savin, T., Eds.; Wiley-Blackwell: Hoboken, NJ, USA, 2011; pp. 157-164.

(C) 2019 by the authors. Licensee MDPI, Basel, Switzerland. This article is an open access article distributed under the terms and conditions of the Creative Commons Attribution (CC BY) license (http://creativecommons.org/licenses/by/4.0/). 